\title{
ON TRAJECTORIES ENTIRELY SITUATED NEAR A HYPERBOLIC SET
}

\author{
D. V. Anosov
}

UDC 517.938

\begin{abstract}
Let $I_{1}$ be a set of points such that their trajectories under a diffeomorphism $f_{1}$ are entirely close enough to a hyperbolic set $F_{1}$ of this diffeomorphism. Then it is proved that the structure of $I_{1}$ and restriction $\left.f_{1}\right|_{I_{1}}$ ("motion in $I_{1}$ ") are essentially defined (up to an equivariant homeomorphism) by "internal dynamics" in $F_{1}$, i.e., by the restriction $\left.f_{1}\right|_{F_{1}}$. (In more detail: the equivariant homeomorphism $g_{1}$ of the set $F_{1}$ on the hyperbolic set $F_{2}$ of the second diffeomorphism $f_{2}$ (probably, acting on another manifold $M_{2}$ ) is extendable to an equivariant homeomorphic embedding $I_{1} \rightarrow M_{2}$. The image of the imbedding contains all the trajectories $f_{2}$ close enough to $F_{2}$.)
\end{abstract}

1. Before formulating the results, let us turn our attention to notation and terminology that we use further.

Let $A$ be a subset of the set $M$. Let us denote by $j_{A}$ an identical embedding of $A$ in $M$, i.e., if $x \in A$, then $j_{A} x$ is the same element $x$, but it is considered as an element of $M$. (This notation will be used in cases where we talk mostly about the same $M$. Therefore, we do not consider $M$ in the notation.)

As before, let $A \subset M$ and let a bijective transformation $f: M \rightarrow M$ act on the set $M$. Let us denote $I(A):=\bigcap_{-\infty}^{\infty} f^{n}(A)$ (for brevity, we will often write shorter: $I A$ ). The notation does not include explicit mention of the dependence $I(A)$ of $f$ because $f$ long remains the same. Obviously, $I(A)$ is a set of points with trajectories (obtained under $f$ ) that are entirely contained in $A$. Also, $I(A)$ is a maximum invariant (with respect to $f$ ) subset of the set $A$ (hence we use the letter $I$ ).

Usually, when we talk about an invariant set, we mean that it is compact, but in this case, generally speaking, $I(A)$ is an exception to the rule. We have defined $I(A)$ without mentioning any topology and we do not need to think about compactness of $I(A)$. Essentially, we did this because in the general context there is a possibility of topology ( $f$ is a homeomorphism of a "good" topological space $M$ ) and there can arise a question about compactness of $I(A)$. But it may happen that $I(A)$ is not compact. However, if $A$ is compact, then $I(A)$ is also compact in this case. Let us note some simple statements

$$
\begin{gathered}
I(A) \subset A, \quad A \subset B \Rightarrow I(A) \subset I(B), \text { more precisely } I(A) \subset A \cap I(B), \\
I(A \cap B)=I(A) \cap I(B), \quad f I(A)=I(A) .
\end{gathered}
$$

Let bijections $f_{i}: M_{i} \rightarrow M_{i}$ act on sets $M_{1}, M_{2}$, and let us define the mapping $\chi: A_{1} \rightarrow M_{2}$ on the subset $A_{1} \subset M_{1}$, where $f_{1} A_{1}=A_{1}$. The mapping $\chi$ is called equivariant (meaning ${ }^{1}$ with respect to $f_{1}$ and $\left.f_{2}\right)$ if $\chi\left(\left.f_{1}\right|_{A_{1}}\right)=f_{2} \chi$. (The condition $f_{1} A_{1}=A_{1}$ guarantees that the left-hand side is defined ${ }^{2}$ on the whole set $A_{1}$. On the right-hand side $f_{2} y$ is defined for all $y \in M_{2}$ including $y=\chi x$.)

Let us notice that from equivariance of $\chi$ there follows the more general formula $\chi\left(\left.f_{1}\right|_{A_{1}}\right)^{n}=f_{2}^{n} \chi$ for all $n \in \mathbb{Z}$. For $n>0$, the formula is proved by an obvious induction on $n$. For $n<0$, to prove the formula we use the relation $\chi\left(\left.f_{1}\right|_{A_{1}}\right)^{-1}=f_{2}^{-1} \chi$. (This means that $x \in A_{1} \Rightarrow \chi f_{1}^{-1} x=f_{2}^{-1} \chi x$, i.e., if $x=f_{1} y$, then $\chi y=f_{2}^{-1} \chi f_{1} y$. In this case, $\chi f_{1} y=f_{2} \chi y$. Therefore, $f_{2}^{-1} \chi f_{1} y=f_{2}^{-1} f_{2} \chi y=\chi y$.)

Translated from Sovremennaya Matematika. Fundamental'nye Napravleniya (Contemporary Mathematics. Fundamental Directions), Vol. 45, Proceedings of the Sixth International Conference on Differential and Functional Differential Equations and International Workshop "Spatio-Temporal Dynamical Systems" (Moscow, Russia, 14-21 August, 2011). Part 1, 2012.

${ }^{1}$ We will drop the last adjustment because $f_{i}$ are fixed hereafter.

${ }^{2}$ It is sufficient to obtain the condition $f_{1} A_{1} \subset A_{1}$. We need the stronger condition $f_{1} A_{1}=A_{1}$ for a more general formula that is mentioned further. This formula contains $f_{i}^{n}$ with different $n$ including negative ones. 
It may occur that $M_{1}=M_{2}, f_{1}=f_{2}$; in this case in the detailed statement (which is usually omitted) we shall speak of "equivariance with respect to $f_{1}$ " instead of "equivariance with respect to $f_{1}$ and $f_{2} . "$

\section{Lemma 1.}

(a) Let $F$ be an invariant (and compact) set of the homeomorphism $f$ of a locally compact space $M$, $U$ its neighborhood in $M$, and $W$ a neighborhood of $F$ in $I(U)$. Then there exists a neighborhood $V \subset U$ of $F$ in $M$ such that $I(V) \subset W$ (in other words, points with trajectories entirely situated close to $F$ are contained in $W$, i.e., in $V)$.

(b) Let $M_{1}, M_{2}$ be locally compact spaces, $f_{i}$ their homeomorphisms on themselves, and $F_{i}$ invariant (with respect to $f_{i}$ ) sets (in $M_{i}$ ) (they are compact by convention). Let $U_{i}$ be neighborhoods of $F_{i}$ in $M_{i}$, and let there exist a continuous equivariant mapping $h: I U_{1} \rightarrow I U_{2}$ that takes $F_{1}$ to $F_{2}$. Therefore, there exists a neighborhood $V_{1}$ of $F_{1}$ in $M_{1}$ such that $V_{1} \subset U_{1}$ for every neighborhood $V_{2}$ of $F_{2}$ in $M_{2}$ (thus, the mapping $h$ is completely defined on $I V_{1}$ ) and $h I V_{1} \subset I V_{2}$.

Proof. (a) (Relatively) open subsets in $I(U)$ are given by $I(U) \cap G$, where $G$ are open subsets in $M$. The neighborhood $W$ of $F$ in $I(U)$ contains a relatively open set that contains $F$. Thus, $W \supset I(U) \cap G$, where $G$ is an open neighborhood of $F$ in $M$. We can always reduce $G$ to a compact neighborhood $V$ of $F$ in $M$ that lies in $U$. Thus, $W \supset I(U) \cap V$, where $V$ is a compact neighborhood of $F$ in $V \subset U$. According to (1), $I(V) \subset I(U) \cap V \subset W$.

(b) $V_{2} \cap I U_{2}$ is a neighborhood of $F_{2}$ in $I U_{2}$ (in the relative topology). From the continuity of $h$ in $I U_{1}$ it follows that there exists a neighborhood $W$ of $F_{1}$ such that $h W \subset V_{2} \cap I U_{2}$. This $W$ contains $V_{1} \cap I U_{1}$, where $V_{1}$ is a neighborhood of $F_{1}$ in $M_{1}$. This means that $h\left(V_{1} \cap I U_{1}\right) \subset V_{2} \cap I U_{2}$. Reducing $V_{1}$ if needed, we can suppose that $V_{1} \subset U_{1}$. Let us prove that

$$
h I V_{1} \subset I V_{2} .
$$

Let us start with a weaker embedding $h I V_{1} \subset V_{2}$. By virtue of (1), $I V_{1} \subset V_{1} \cap I U_{1}$. Therefore, $h I V_{1} \subset h\left(V_{1} \cap I U_{1}\right) \subset V_{2} \cap I U_{2} \subset V_{2}$. We obtain the stronger embedding (2) using the equivariance of $h$. We want to prove that if $y \in h_{1} I V_{1}$ (i.e., $y=h_{1} x$ with some $x \in I V_{1}$ ), then all $f_{2}^{n} y \in V_{2}$. But $f_{2}^{n} y=f_{2}^{n} h_{1} x=h_{1} f_{1}^{n} x$ and $x \in I V_{1}$. Hence, all $f_{1}^{n} x \in I V_{1}$ and we obtain that $f_{2}^{n} y=h x^{\prime}$ with some $x^{\prime} \in I V_{1}$, i.e., $f_{2}^{n} y \in h I V_{1} \subset V_{2}$.

Remark 1. Let us denote by $\mathcal{O}$ the system of all neighborhoods of $F$ in $U$, and by $\mathcal{B}$ its basis. According to Lemma 1, (a) there exists an analogy between $\mathcal{J}=\{I V: V \in \mathcal{O}\}$ and $\mathcal{B}$. Any $W \in \mathcal{O}$ contains some $V \in \mathcal{J}$ and some $V_{1} \in \mathcal{B}$. We can find the same analogy in Lemma 1, (b). Now, let us have neighborhoods in $M_{1}$ and in $M_{2}$. Let us denote by $\mathcal{O}_{i}$ a system of neighborhoods of $F_{i}$ in $U_{i}$, by $\mathcal{B}_{i}$ one of its bases, and by $\mathcal{J}_{i}$ the system of sets $\left\{I V: V \in \mathcal{O}_{i}\right\}$. If the mapping $h: U_{1} \rightarrow U_{2}$ is continuous, then

$$
\left.\forall W_{2} \in \mathcal{O}_{2}\left(\text { or } \in \mathcal{B}_{2}\right) \quad \exists W_{1} \in \mathcal{O}_{1} \text { (or } \in \mathcal{B}_{1}\right) \quad h W_{1} \subset W_{2} .
$$

From Lemma 1, (b) it follows that if the mapping $h: I U_{1} \rightarrow I U_{2}$ is continuous, then

$$
\forall W_{2} \in \mathcal{J}_{2} \quad \exists W_{1} \in \mathcal{J}_{1} \quad h W_{1} \subset W_{2} .
$$

But, generally speaking, sets of type $I(V)$ do not form a basis of the neighborhoods of $F$ in $I(U)$. For example, let $M=\mathbf{S}^{1} \subset \mathbb{C}$ (a unit circle on the complex plane), the mapping $f: \mathbf{S}^{1} \rightarrow \mathbf{S}^{1}$ takes \pm 1 into $\pm 1, f\left(e^{i \varphi}\right)$ equals $e^{i \psi}$ for $0<|\varphi|<\pi$ with some $\psi$ such that $0<|\psi|<|\varphi|$, and $F=\{1\}$. If $U=\mathbf{S}^{1}$ or $U=\mathbf{S}^{1} \backslash\{-1\}$; then $I(U)=U$. If we obtain $V$ by removing just two points from $\mathbf{S}^{1}$ or by removing just one point from $\mathbf{S}^{1} \backslash\{-1\}$, then $I(V)$ is not a neighborhood of $F$ in $I(U)$. Hence, there exists at least one point $x$ of type $e^{i \varphi}$ with $0<|\varphi|<\pi$ outside $V$. It means that $x \notin I(V)$. Therefore, all $f^{n}(x) \notin I(V)$ and $f^{n}(x) \rightarrow 1$ as $n \rightarrow \infty$. Let us notice that if $V \subset\left\{e^{i \varphi}:|\varphi|<\pi-\varepsilon\right\}$ with some $\varepsilon>0$, then $I(V)=F$. 
In what follows, we will consider $M, M_{1}$, and $M_{2}$ as smooth manifolds with metrics $\rho$ (this letter will denote different metrics on different manifolds). By $U_{\delta}(A)$ let us denote a $\delta$-neighborhood of the set $A$. This neighborhood is closed.

By "mapping" we will mean a "continuous mapping," with the exception of the case where a mapping is built anew. Then we cannot assume its continuity but rather verify it.

2. The main theorem of this work refers ${ }^{3}$ to the case where we consider the hyperbolic sets $F_{1}$ and $F_{2}$ of self-diffeomorphisms $f_{1}$ and $f_{2}$ of smooth manifolds $M_{1}$ and $M_{2}$. The theorem affirms that the construction of the set of points with trajectories close enough to $F_{1}$, i.e., sets $I\left(V_{1}\right)$, where $V_{1}$ is a sufficiently small neighborhood of $F_{1}$, and the motions inside the set are defined by "internal dynamics" in $F_{1}$, i.e., the homeomorphism $\left.f_{1}\right|_{F_{1}}$ with its iteration (with iteration of $\left.f_{1}\right|_{F_{1}} ^{-1}$ ) provides the dynamical system $\left(I\left(V_{1}\right),\left\{\left.f_{1}\right|_{I\left(V_{1}\right)} ^{n}\right\}\right)$. We have mentioned just $M_{1}, f_{1}, F_{1}$ (and $V_{1}$ ), but we also need $M_{2}, f_{2}, F_{2}$ (and some neighborhood $V_{2}$ of $F_{2}$ ). If the second diffeomorphism $f_{2}$ (acting, generally speaking, on another manifold $M_{2}$ ) has a hyperbolic set $F_{2}$ with the same "internal dynamics," then the set $I\left(V_{2}\right)$ of points from $M_{2}$ with trajectories entirely situated close enough to $F_{2}$ in some sense is constructed in the same way as $I\left(V_{1}\right)$. The "internal dynamics" in $I\left(V_{1}\right)$ is constructed in the same sense as "internal dynamics" in $I\left(V_{2}\right)$. The coincidence of "internal dynamics" in $F_{1}$ and $F_{2}$ means that there exists an equivariant homeomorphism $g_{1}: F_{1} \stackrel{\text { onto }}{\longrightarrow} F_{2}$, i.e, the homeomorphism $F_{1} \stackrel{\text { on }}{\longrightarrow} F_{2}$ that conjugates $\left.f_{1}\right|_{F_{1}}$ and $\left.f_{2}\right|_{F_{2}}: g_{1} \circ\left(\left.f_{1}\right|_{F_{1}}\right)=f_{2} \circ g_{1}$. (In the same sense the inverse homeomorphism $g_{2}=g_{1}^{-1}$ takes the second system into the first one.)

The main theorem affirms something about the points with trajectories entirely close to $F_{i}$. Obviously, these points belong to $I\left(V_{i}\right)$, where $V_{i}$ are sufficiently small neighborhoods of $F_{i}$, and motions in the sets with these points form the dynamic system $\left\{\left(\left.f_{i}\right|_{I\left(V_{i}\right)}\right)^{n}\right\}$. We can naturally compare these systems by an equivariant homeomorphism, considering the two systems $\left\{\left(I\left(V_{i}\right),\left(\left.f_{i}\right|_{I\left(V_{i}\right)}\right)^{n}\right)\right\}$ "the same" if $\left.f_{i}\right|_{I\left(V_{i}\right)}$ are conjugated with each other by a homeomorphism $h: I\left(V_{1}\right) \rightarrow I\left(V_{2}\right)$, i.e., if there exists an equivariant homeomorphism $I\left(V_{1}\right) \stackrel{\text { onto }}{\longrightarrow} I\left(V_{2}\right)$. But we could just prove the statement "about likeness" of these dynamic systems in a weaker sense. ${ }^{4}$

Theorem 1. Under our assumptions there exist such neighborhoods $V_{i}^{\prime} \subset V_{i}$ of $F_{i}$ and such equivariant injections ${ }^{5} h_{1}: I\left(V_{1}\right) \rightarrow I\left(M_{2}\right), h_{2}: I\left(V_{2}\right) \rightarrow I\left(M_{1}\right)$ that

$$
\left.\begin{array}{ll}
h_{1} I\left(V_{1}^{\prime}\right) \subset I\left(V_{2}\right), & h_{2} I\left(V_{2}^{\prime}\right) \subset I\left(V_{1}\right), \\
I\left(V_{2}^{\prime}\right) \subset h_{1} I\left(V_{1}\right), & I\left(V_{1}^{\prime}\right) \subset h_{2} I\left(V_{2}\right),
\end{array}\right\}
$$

(The neighborhoods can be considered compact.)

Let us notice that $I\left(V_{i}\right)$ contains compact subsets $A_{i}$ such that $A_{i} \supset I\left(V_{i}^{\prime}\right)$ for some neighborhoods $V_{i}^{\prime}$ of $F_{i}$. Also, we obtain the inverse homeomorphisms $h_{1}: A_{1} \rightarrow A_{2}, h_{2}: A_{2} \rightarrow A_{1}$ by restriction to mappings $h_{i}$ on $A_{i}$. For example, let $A_{1}=I\left(V_{1}^{\prime}\right)\left(V_{i}^{\prime}\right.$ and $V_{i}$ here and further are the same as in the statement of the theorem) and $h_{1} A_{1}=A_{2}$. Thus, if $a_{1} \in A_{1}$ then $h_{1} a_{1} \in I\left(V_{1}\right), h_{2} h_{1}$ is defined (according to the first formula in (3)) and $h_{2} h_{1} a_{1}=a_{1}$ (according to the first formula in (4)). But if $a_{2} \in A_{2}$, then $a_{2}=h_{1} a_{1}$ with some $a_{1} \in A_{1}$. Then $h_{2} a_{2}=h_{2} h_{1} a_{1}=a_{1} \in A_{1} \subset I\left(V_{1}\right)$ (according to the first formula in (4)), i.e., $h_{1} h_{2} a_{2}$ is defined and $h_{1} h_{2} a_{2}=h_{1}\left(h_{2} h_{1} a_{1}\right)=h_{1} a_{1}$ (according to the first formula in (4)), i.e., $h_{1} h_{2} a_{2}=a_{2}$.

\footnotetext{
${ }^{3}$ Actually, the theorem has a "semilocal" character. The theorem is concerned only with what happens close to $F_{i}$. Therefore we need only that functions $f_{i}: U_{i} \rightarrow M_{i}$ with necessary characteristics be defined in some neighborhoods $U_{i}$ of $F_{i}$. However, the full formulation of all these characteristics is rather cumbersome, though the "semilocal" statement is formulated as one that is concerned with the self-diffeomorphism of the whole phase manifold in the theory of smooth dynamical systems

${ }^{4}$ It recalls a weak isomorphism in the ergodic theory. But is it important?

${ }^{5}$ The equivariant injections $h_{1}: I\left(V_{1}\right) \rightarrow I\left(M_{2}\right)$, and $h_{1}: I\left(V_{1}\right) \rightarrow M_{2}$ are equal because $I\left(M_{2}\right)=M_{2}$.
} 
It can be proved that $A_{2}$ contains $I\left(W_{2}\right)$, where $W_{2}$ is a neighborhood in $F_{2}$. (We can obtain this in the same way as we did with the third formula in (3).) But it is unclear if we can obtain (with some modifications in constructions) that each $A_{i}$ coincides with some $I\left(W_{i}\right)$, where $W_{i}$ are some neighborhoods of $F_{i}$.

3. Remark 2. In the statement of Theorem 1 we speak of one set $V_{1}$, one set $V_{2}$, one $V_{1}^{\prime}$, and one $V_{2}^{\prime}$. But it can happen that some of the sets $V_{i}, V_{i}^{\prime}$ or even all of them are "small enough," where the "required order of smallness" can change from case to case. It means that small neighborhoods should be contained in the prescribed neighborhoods of $F_{i}$. Thus, we consider that the neighborhoods $\bar{V}_{1}, \bar{V}_{1}^{\prime}$ of $F_{1}$ and neighborhoods $\bar{V}_{2}, \bar{V}_{2}^{\prime}$ of $F_{2}$ are given. We want to know if there exist neighborhoods $\widehat{V}_{1}, \widehat{V}_{1}^{\prime}$ of $F_{1}$ and neighborhoods $\widehat{V}_{2}, \widehat{V}_{2}^{\prime}$ of $F_{2}$ such that

$$
\widehat{V}_{1} \subset V_{1} \cap \bar{V}_{1}, \quad \widehat{V}_{2} \subset V_{2} \cap \bar{V}_{2}, \quad \widehat{V}_{1}^{\prime} \subset V_{1}^{\prime} \cap \bar{V}_{1}^{\prime}, \quad \widehat{V}_{2}^{\prime} \subset V_{2}^{\prime} \cap \bar{V}_{2}^{\prime}, \quad \widehat{V}_{1}^{\prime} \subset \widehat{V}_{1}, \quad \widehat{V}_{2}^{\prime} \subset \widehat{V_{2}}
$$

(relation (5), in particular, guarantees that mappings $h_{i}$ are completely defined on $\widehat{V}_{i}$ and all the more on $\widehat{V}_{i}^{\prime}$ ) and that

$$
\begin{array}{cl}
h_{1} I \widehat{V}_{1}^{\prime} \subset I \widehat{V}_{2}, & I \widehat{V}_{1}^{\prime} \subset h_{2} I \widehat{V}_{2}, \\
h_{2} I \widehat{V}_{2}^{\prime} \subset I \widehat{V}_{1}, & I \widehat{V}_{2}^{\prime} \subset h_{1} I \widehat{V}_{1}, \\
\left.h_{2} h_{1}\right|_{I \widehat{V}_{1}^{\prime}}=j_{I \widehat{V}_{1}^{\prime}}, & \left.h_{1} h_{2}\right|_{I \widehat{V}_{2}^{\prime}}=j_{I \widehat{V}_{2}^{\prime}}
\end{array}
$$

The new ("hatted") neighborhoods should be obtained by reducing the old neighborhoods. The question is if we can do this reducing in the way that relations (3), (4) could be replaced by the similar relations but with "hatted" neighborhoods? And now let us substitute $h_{i}$ by $\widehat{h}_{i}=\left.h_{i}\right|_{I \widehat{V}_{i}}$ and relations (6)-(8) by

$$
\begin{array}{cc}
\widehat{h}_{1} I \widehat{V}_{1}^{\prime} \subset I \widehat{V}_{2}, & I \widehat{V}_{1}^{\prime} \subset \widehat{h}_{2} I \widehat{V}_{2}, \\
\widehat{h}_{2} I \widehat{V}_{2}^{\prime} \subset I \widehat{V}_{1}, & I \widehat{V}_{2}^{\prime} \subset \widehat{h}_{1} I \widehat{V}_{1}, \\
\left.\widehat{h}_{2} \widehat{h}_{1}\right|_{I \widehat{V}_{1}^{\prime}}=j_{I \widehat{V}_{1}^{\prime}}, & \left.\widehat{h}_{1} \widehat{h}_{2}\right|_{I \widehat{V}_{2}^{\prime}}=j_{I \widehat{V}_{2}^{\prime}}
\end{array}
$$

This transition from (6)-(8) to (9)-(11) looks like changing the notation. But we should take into account that the domain of definition of $\widehat{h}_{i}$ is narrowed down compared with that of $h_{i}$. Therefore, it is unclear if the action of $\widehat{h}_{i}$ is defined on any set where $h_{i}$ acts. It is also unclear if the composition of mappings $\widehat{h}_{i}$ is defined under the condition that the same composition of mappings $h_{i}$ is defined. Specifically, does the following type of relations from (9)-(11) make sense?

$$
\widehat{h}_{1} I \widehat{V}_{1}^{\prime}, \quad \widehat{h}_{2} I \widehat{V}_{2},\left.\quad \widehat{h}_{2} \widehat{h}_{1}\right|_{I \widehat{V}_{1}^{\prime}}
$$

The answers are positive. We take Theorem 1 on trust (delaying the proof until Sec. 4) in this section. Firstly, we discuss how to pass from Theorem 1 to (6)-(8) (in this transition the mappings $h_{i}$ do not change, but the domains $V_{i}, V_{i}^{\prime}$ are reduced to $\left.\widehat{V}_{i}, \widehat{V}_{i}^{\prime}\right)$. Then we discuss the transition from (6)-(8) to (9)-(11) (in this transition the mappings change, namely, their domains of definition are narrowed down but the domains of the mapping $\widehat{V}_{i}, \widehat{V}_{i}^{\prime}$ do not change). Let us go into greater details. Formally, we do not have to use $\widehat{h}_{i}$ and (9)-(11), but we reduce the domains of definition of our mappings.

First, we turn our attention to (6)-(8).

Let us denote by $\widehat{V}_{i}$ the neighborhoods of $F_{i}$ that satisfy the first two embeddings in (5) (for the sake of definiteness, let us take $\widehat{V}_{i}=V_{i} \cap \bar{V}_{i}$ ). Thereafter, we consider (6) as two conditions that are imposed on $\widehat{V}_{1}^{\prime}$ and (7) as two conditions that are imposed on $\widehat{V}_{2}^{\prime}$. These conditions are "conditions of smallness." If the neighborhood $\widehat{V}_{i}^{\prime}$ satisfies the corresponding condition, then the contraction of the 
neighborhood does not change the fulfilment of the condition. The cases where $i=1,2$ are similar. Therefore, let us consider the first case.

Although relations (6) with fixed $I \widehat{V}_{2}$ look like two conditions on $\widehat{V}_{1}^{\prime}$, it is sufficient to find $\widehat{V}_{1}^{\prime}$ that satisfies the first condition

$$
h_{1} I \widehat{V}_{1}^{\prime} \subset I \widehat{V}_{2}
$$

(and, also, the conditions of smallness for $\widehat{V}_{1}^{\prime}$ that are the third and the fourth conditions in (5)). The action of mapping $h_{2}$ is defined on $I \widehat{V}_{2}$ and on $h_{1} I \widehat{V}_{1}^{\prime}$. Applying the mapping $h_{2}$ to both sides of (13), we obtain $h_{2} h_{1} I \widehat{V}_{1}^{\prime} \subset h_{2} I \widehat{V}_{2}$. But the mapping $h_{2} h_{1}$ is the identity mapping on the set $I V_{1}^{\prime}$ and $\widehat{V}_{1}^{\prime}$ is a part of the last one (because of the third relation in (5)). Hence, $I \widehat{V}_{1}^{\prime} \subset h_{2} I \widehat{V}_{2}$, and this is the second relation in (6).

It remains to check the existence of the neighborhood $\widehat{V}_{1}$ of $F_{1}$ in $M_{1}$ such that the conditions of smallness are fulfilled, i.e., the third and the fourth conditions in (5) and the first condition in (6). Let us apply Lemma 1, (b) denoting neighborhoods $V_{1}^{\prime}$ and $V_{2}$ of the sets $F_{1}$ and $F_{2}$ by $U_{1}$ and $U_{2}$, the mapping $h_{1}: I V_{1}^{\prime} \rightarrow I V_{2}$ by $h$, and the neighborhood $\widehat{V}_{2}$ of the set $F_{2}$ in $M_{2}$ by $V_{2}$. The lemma guarantees the existence of such neighborhood of the set $F_{1}$. The neighborhood was denoted by $V_{1}$ in the lemma, but now we denote it by $\widetilde{V}_{1}^{\prime}$. This means that in place of $V_{1} \subset U_{1}$ and $h I V_{1} \subset I V_{2}$ (in Lemma) now we have $\widehat{V}_{1}^{\prime} \subset V_{1}^{\prime}$ and $h_{1} I \widehat{V}_{1}^{\prime} \subset I \widehat{V}_{2}$. The last embedding coincides with the first relation in (6).

We have not only checked that expression $h_{1} I V_{1}$ makes sense, but proved the first formula (6). The second one was already proved above; thus, now both relations (6) are proved. And since relations (7) are similar (6), it remains to check two relations (8) from the whole group of formulas under discussion (6)-(8). These are again analogs of each other, and we restrict ourselves to checking the first one. Denoting for brevity $k=\left.h_{1}\right|_{I V_{1}^{\prime}}$, rewrite the first relation (8) in the form $h_{2}\left(\left.k\right|_{I V_{1}^{\prime}}\right)=j_{I V_{1}^{\prime}}$. The domain of $h_{2} k$ as well as that of $k$ is $I V_{1}^{\prime}$; narrowing it down to $I \widehat{V}_{1}^{\prime}$, from $h_{2} k$ we get a mapping $\left.\left(h_{2} k\right)\right|_{I \widehat{V}_{1}^{\prime}}$, which coincides with $h_{2}\left(\left.k\right|_{I \widehat{V}_{1}^{\prime}}\right)$. From $j_{I V_{1}^{\prime}}$ we get a mapping $\left.j_{I V_{1}^{\prime}}\right|_{I \widehat{V}_{1}^{\prime}}=j_{I \widehat{V}_{1}^{\prime}}$. We come to relation $h_{2}\left(\left.k\right|_{I \widehat{V}_{1}^{\prime}}\right)=j_{I \widehat{V}_{1}^{\prime}}$, which coincides with the first relation (8).

Now we pass to the group of formulas (9)-(11). Since the domains of $\widehat{h}_{i}$ are narrowed down with respect to those of $h_{i}$, while passing from (6)-(8) to (9)-(11) the following questions arise. Are actions $\widehat{h}_{i}$ that appear in (9)-(11) defined in $I \widehat{V}_{i}, I \widehat{V}_{i}^{\prime}$ ? If they are, so that all objects defined in (9)-(11) are defined correctly, are some of them really parts of the others, as stated in the same formulas?

The answers to all these questions are also positive. By the definition of mappings $\widehat{h}_{i}$, they are defined on $I \widehat{V}_{i}$, and hence on smaller sets $I \widehat{V}_{i}^{\prime}$. In (11) appear compositions of $\widehat{h}_{2} \varphi$ with some mappings $\varphi$. Such compositions are defined if

$$
\text { the range of } \varphi \subset \text { the domain of } \widehat{h}_{2} \text {. }
$$

We have $\varphi=\left.\widehat{h}_{1}\right|_{I \widehat{V}_{1}^{\prime}}$. Its range is $\widehat{h}_{1}\left(I \widehat{V}_{1}^{\prime}\right)=h_{1}\left(I \widehat{V}_{1}^{\prime}\right) \subset I \widehat{V}_{2}$, that is, the domain of $\widehat{h}_{2}$. Hence, (14) holds.

Finally, wherever the mapping $\widehat{h}_{i}$ is defined, it coincides with $h_{i}$. And since in (9)-(11) the action $\widehat{h}_{i}$ on these objects is defined everywhere, in these formulas $\widehat{h}_{i}$ can be everywhere replaced with $h_{i}$. Then we obtain formulas (6)-(8), which were checked above.

4. We prove directly two other theorems, which imply Theorem 1.

Theorem 2. Under assumptions of Theorem 1 there exists a neighborhood $V_{1}$ of the set $F_{1}$ and an equivariant mapping $h_{1}: I\left(V_{1}\right) \rightarrow M_{2}$ that extends $g_{1}$.

Changing the roles of $M_{1}, f_{1}, F_{1}, g_{1}$ and $M_{2}, f_{2}, F_{2}, g_{2}$, we conclude that there exist a neighborhood $V_{2}$ of the set $F_{2}$ and an equivariant mapping $h_{2}: I\left(V_{2}\right) \rightarrow M_{1}$ that extends $g_{2}$. 
Theorem 3 is formulated for the situation where we deal with a single hyperbolic set $F$ of the diffeomorphism $f$ of the manifold $M$ onto itself. (Further we use these theorems in the situation described at the beginning of Sec. 2, applying them both to $M=M_{1}, f=f_{1}$, and $F=F_{1}$ and to $M=M_{2}, f=f_{2}$, and $F=F_{2}$.) We adopt a convention to mark often, though not always, those objects that are (more directly in some sense) related to $F_{i}$, by a subscript $i$ (for example, ${ }^{6}$ it is the case for $V_{i}$ in Theorem 1). For the sake of brevity, sometimes we mention "a two- $F_{i}$ situation" and "a one- $F$ situation." While mentioning these situations, we implicitly refer to the fact that their presence implies the validity of two statements. One of these is the theorem about families of pseudotrajectories given in Sec. 6. The other one tells us that a hyperbolic set $F$ has a neighborhood $S$ in $M$ where motions are separated (namely, the letter $S$ stays for separation). The respective separation constant is denoted by $D$, and thus if $x, y \in I(S)$ and $\rho\left(f^{n} y, f^{n} x\right) \leq D$ for all $n$, then $x=y$. Evidently, there exists a $R>0$ such that the closed $R$-neighborhood $U_{R}(F)$ of the set $F$ in $M$ is compact and $U_{R}(F) \subset S$. (Notations $S, R$, and $D$ introduced now are used further without extra comments. The same can be said about the two- $F$ situation - whenever our arguments have to do with $F_{i}$, we write $S_{i}, R_{i}, D_{i}$.)

Theorem 3. Let $U$ (in the one-F situation) be a compact neighborhood of $F$ in $M$, and let $\varphi: I(U) \rightarrow$ $M$ be an equivariant mapping such that $\left.\varphi\right|_{F}=j_{F}$. Then $F$ has a neighborhood $V \subset U$ such that $\left.\varphi\right|_{I(V)}=j_{I(V)}$.

In Sec. 6, we explain how to use Theorem 1 and Lemma 1 in order to obtain the already known $[3,5]$ result on the intrinsic character of properties such as local maximality and local premaximality in the class of hyperbolic sets. First we give some information on local premaximality, starting with the definition of this property, since these data are mostly new and not yet treated in textbooks and reviews. (While local maximality has attracted attention rather long ago and is considered in textbooks $[7,10]$, discussing it would make a paper like this too detailed.)

Then in Sec. 7, Theorem 1 is deduced from Theorems 2 and 3. In the arguments of Secs. 6 and 7, we basically do not use ideas that contain more or less novel elements - such a new element is contained in the theorems we apply, but they are used, so to say, in a rather "straightforward" way.

Finally, in Secs. 8 and 9, Theorem 2 is proved, ${ }^{7}$ and in Sec. 10, so is Theorem 3. To my mind, the arguments of Secs. $8-10$ are the most novel ones. ${ }^{8}$

5. In mid-60's the question arose whether any hyperbolic set $F$ has the following property: Every neighborhood of $F$ contains a locally maximal hyperbolic set such that $F$ is a part of it. Then a positive answer was expected, but in the mid-80's Fati gave an example that provided the negative answer to this question. For some reason, the Fati example was not published, but the same one (to our understanding) was rediscovered and published by Crovisier [6]. As long as there was hope that all hyperbolic sets have a certain property, no special term seemed necessary for it. But such necessity was felt when it turned out that some hyperbolic sets do have this property while others do not have it. In this connection the author suggested to call this property local premaximality.

In the Fati-Crovisier example the hyperbolic set that is not locally premaximal is two-dimensional. Fisher constructed an example of a one-dimensional hyperbolic set that is not locally premaximal. ${ }^{9}$

\footnotetext{
${ }^{6}$ This convention does not work if an object is related to both $F_{i}$ at the same time. For example, such is $g_{1}$ in Theorem 1.

${ }^{7} \mathrm{~A}$ part of the proof of Theorem 2 is given with respect to the one- $F$ situation. This part is the subject of Sec. 8 . But Theorem 2 itself by its very formulation deals with the two $F$ situation, and, naturally, the same situation occurs when we complete the proof of this theorem, which is the subject of Sec. 9.

${ }^{8}$ With a clause that similar arguments were briefly hinted at in $[2,3]$.

${ }^{9}$ The main example from [8] (with some simple modification) was discussed in [4]. In particular, there were formulated some "intrinsic" conditions that guarantee lack of local premaximality appropriate to these examples. A more recent general theorem [3, 5] overlaps with observations contained in [4] that the lack of local premaximality in these examples is connected with "intrinsic" peculiarities of the behavior of trajectories. But it is possible that these peculiarities are to some extent interesting as such.
} 
Using his example, one can easily obtain examples of hyperbolic sets of any positive dimension that are not locally premaximal. As for hyperbolic sets of dimension zero, the author has proved that they are locally premaximal [2]. (Maybe hopes for local premaximality of all hyperbolic sets had to do with excessive attention to the zero dimensional case.) Another class of locally premaximal hyperbolic sets is given by Fisher. It is the class of topologically transitive hyperbolic subsets of surfaces [9]. At the same time topological transitivity of a hyperbolic subset as such does not guarantee its local premaximality. In the respective example the phase space is four-dimensional [8].

In [3] we prove the "intrinsic" character of the property of local premaximality in the class of hyperbolic sets: Its presence or absence in a hyperbolic set $F$ depend only on the action of the diffeomorphism $f$ under consideration on the set $F$ (i.e., so to speak, on the properties of "internal dynamics" in $F$, that is, of the dynamical system $\left(\left(\left.f\right|_{F}\right)^{n} ; n \in \mathbb{Z}\right)$ with a phase space $\left.F\right)$. (But the explicit formulation of this internal set (which is equivalent to local premaximality) is unknown). In Sec. 6, we explain that this theorem easily follows from the results of the present paper. The same holds for local maximality, but it is a matter of no special interest and is mentioned in Sec. 6 only for the sake of completeness. In fact, for local maximality there is a complete formulation of an equivalent "intrinsic" property (in the class of hyperbolic sets) independent of this paper: namely, it consists in "internal tracing of internal pseudo-trajectories."

6. Here we explain how the statements on intrinsic character of properties of local maximality and of local premaximality in the class of hyperbolic sets immediately follow from Theorem 1 and Lemma 1.

In the notation of Theorem 1 , let a hyperbolic set $F_{1}$ be locally maximal, i.e., it has a neighborhood $W_{1}$ in $M_{1}$ such that $I W_{1}=F_{1}$. Reducing $W_{1}$ if necessary, one can assume that $W_{1} \subset V_{1}^{\prime}$. There exists a neighborhood $W_{2}$ of the set $F_{2}$ in $M_{2}$ such that $W_{2} \subset V_{2}^{\prime}$ and $h_{2} I W_{2} \subset I W_{1}$. In our case it means that $h_{2} I W_{2}=F_{1}$. Therefore, $I W_{2}=h_{1} h_{2} I W_{2}=h_{1} F_{1}=F_{2}$.

Now let a hyperbolic set $F_{1}$ be locally premaximal, i.e., such that each of its neighborhood $W_{1}$ (in $M_{1}$ ) contains a locally maximal set containing $F_{1}$. We should check that any neighborhood $W_{2}$ of the set $F_{2}$ in $M_{2}$ has the same property. Reducing $W_{2}$ if necessary, we can assume that $W_{2} \subset V_{2}^{\prime}$ and that the set $I W_{2}$ is hyperbolic (it is known that an invariant set which is close enough to a hyperbolic set is hyperbolic itself). By Lemma $1, F_{1}$ has in $M_{1}$ a neighborhood $W_{1} \subset V_{1}^{\prime}$ such that $h_{1} I W_{1} \subset I W_{2} \subset W_{2}$. And due to local premaximality of $F_{1}$, it has a neighborhood $W_{1}^{\prime} \subset W_{1}$ in $M_{1}$ such that $I W_{1}^{\prime}$ is locally maximal. We have

$$
h_{1} I W_{1}^{\prime} \subset h_{1} I W_{1} \subset I W_{2} \subset W_{2} \subset V_{2}^{\prime} .
$$

Hence the mapping $h_{2}$ is defined on $h_{1} I W_{1}^{\prime}$. Similarly, $h_{1}$ is defined on $h_{2} I W_{2}^{\prime}$, where $W_{2}^{\prime}$ is a neighborhood of $F_{2}$ in $M_{2}$ with properties similar to those of $W_{1}^{\prime}$. Further we note that mappings

$$
\left.h_{1}\right|_{I\left(V_{1}^{\prime}\right)}: I\left(V_{1}^{\prime}\right) \rightarrow h_{1} I\left(V_{1}^{\prime}\right),\left.\quad h_{2}\right|_{I\left(V_{2}^{\prime}\right)}: I\left(V_{2}^{\prime}\right) \rightarrow h_{2} I\left(V_{2}^{\prime}\right),
$$

are equihomeomorphisms. (Equivariance of $h_{i}$ takes place even on larger sets $I\left(V_{i}\right)$, and mappings $(15)$ have continuous inverse ones $\left.h_{2}\right|_{h_{1} I\left(V_{1}^{\prime}\right)},\left.h_{1}\right|_{h_{2} I\left(V_{2}^{\prime}\right)}$.) At the same time, the set $h_{1} I\left(W_{1}^{\prime}\right)$ is contained in the hyperbolic set $I W_{2}$ and therefore is hyperbolic itself. It turns out that $h_{1} I\left(W_{1}^{\prime}\right)$ is a hyperbolic set that is equihomeomorph (by $\left.h_{1}\right|_{I W_{1}^{\prime}}$ ) to a locally maximal set $\left(I W_{1}^{\prime}\right)$, therefore, the set $h_{1} I\left(W_{1}^{\prime}\right)$ is also locally maximal.

7. Here we see that Theorem 1 follows from Theorems 2 and 3. First of all, by Theorem 2, there exist a neighborhood $V_{1}$ of $F_{1}$ and an equivariant mapping $h_{1}: I\left(V_{1}\right) \rightarrow M_{2}$ that extends $g_{1}$. Similarly, there exist a neighborhood $V_{2}$ of $F_{2}$ and an equivariant mapping $h_{2}: I\left(V_{2}\right) \rightarrow M_{1}$ that extends $g_{2}$. Since $h_{1} F_{1}=F_{2}$, the image $h_{1}\left(W_{1}\right)$ of some neighborhood $W_{1}$ of $F_{1}$ in $I\left(V_{1}\right)$ is contained in $V_{2}$. By Lemma 1 (with a slight change of notation), in $M_{1}$ there is a neighborhood $U_{1}$ of set $F_{1}$ such that $U_{1} \subset V_{1}, I\left(U_{1}\right) \subset W_{1}$, and $h_{1} I\left(U_{1}\right) \subset V_{2}$. Hence, an equivariant mapping $\varphi:=\left.h_{2} h_{1}\right|_{I\left(U_{1}\right)}: I\left(U_{1}\right) \rightarrow M_{1}$ is defined on $I\left(U_{1}\right)$, and it coincides with $j_{F_{1}}$ on $F_{1}$. By Theorem 3 , the set $F_{1}$ has a neighborhood $V_{1}^{\prime} \subset U_{1}$ in $M_{1}$ such that $\left.h_{2} h_{1}\right|_{I\left(V_{1}^{\prime}\right)}=\left.\varphi\right|_{I\left(V_{1}^{\prime}\right)}=j_{I\left(V_{1}^{\prime}\right)}$. Inclusions $V_{1}^{\prime} \subset U_{1}$ and $h_{1} I\left(U_{1}\right) \subset V_{2}$ guarantee 
the validity of the first inclusion (3). Similarly, in $M_{2}$ there exists a neighborhood $U_{2}$ of $F_{2}$ such that $U_{2} \subset V_{2}, h_{2} I\left(U_{2}\right) \subset V_{1}$, and a neighborhood $V_{2}^{\prime} \subset U_{2}$ such that $\left.h_{1} h_{2}\right|_{I\left(V_{1}^{\prime}\right)}=\left.j\right|_{I\left(V_{1}^{\prime}\right)}$. In this case $h_{2} I\left(V_{2}^{\prime}\right) \subset h_{2} I\left(U_{2}\right) \subset I\left(V_{1}\right)$ is the second inclusion (3). It remains to check the validity of two last inclusions in (3). Using first the second inclusion (4), and then the second inclusion (3), we get: $I\left(V_{2}^{\prime}\right)=h_{1} h_{2} I\left(V_{2}^{\prime}\right) \subset h_{1} I\left(V_{1}\right)$, i.e., the third inclusion (3). Similarly, the fourth inclusion can be proved using the first inclusion (4) and the first inclusion (3).

8. We now start the proof of Theorem 2. Its main idea is as follows. Let the trajectory $\left\{f^{n} x\right\}$ of a point $x$ be completely situated near $F$, say, $x \in I\left(U_{r}\left(F_{1}\right)\right)$. We approximate this trajectory "up to $r$ " with a sequence of points $y=\left(y_{n} ; n \in \mathbb{Z}\right)$ that lie in $F_{1}$; in other words, $y_{n} \in F_{1}$ are such that

$$
\rho\left(y_{n}, f^{n} x\right) \leq r \text { for all } n \in \mathbb{Z} .
$$

Generally speaking, the sequence $y$ is not a trajectory, but it is similar to one in the sense that $f y_{n} \approx y_{n+1}$. If

$$
\text { all } \rho\left(y_{n+1}, f y_{n}\right) \leq \delta,
$$

then we call the sequence $y$ a $\delta$-pseudo-trajectory ${ }^{10}$.

Our sequence $y$ satisfies (16) from the very beginning, and (17) was not mentioned; but it is easy to establish (this will be done accurately below) that for $r$ small enough inequalities (17) hold with arbitrarily small $\delta>0$.

Since points $y_{n}$ that make the sequence $y$ were taken in $F_{1}$, we can apply to them the mapping $g_{1}: F_{1} \rightarrow F_{2}$. Thus we get a sequence $z=\left(z_{n}\right)$ of points $z_{n}=g_{1} y_{n}$ lying in $F_{2}$. It is a $\Delta$-pseudotrajectory, where $\Delta$ depends on $\delta$, and for $\delta$ small enough (that is, for $r$ small enough), $\Delta$ is also small. We arrive at $\Delta$-pseudo-trajectories in the hyperbolic set $F_{2}$. For a long time it is known that for $\Delta$ small enough there is a real trajectory near such a pseudo-trajectory ${ }^{11}\left\{f_{2}^{n} u\right\}$ (the exact formulation in the form we need is given below).

The correspondence $x \mapsto u$ is the desired mapping $h_{1}: I\left(U_{r}\right) \rightarrow M_{2}$. We explain its equivariance, i.e., the fact that application of $h_{1}$ to $f_{1}^{k} x$ gives $f_{2}^{k} u$. In fact, we assign to the point $f_{1}^{k} x$ :

- first, the sequence $\tilde{y}=\left(\tilde{y}_{n}\right)=\left(y_{n+k} ; n \in \mathbb{Z}\right)$;

- then, the sequence $\tilde{z}=\left(\tilde{z}_{n}\right)$, where $\tilde{z}_{n}=g_{1} \tilde{y}_{n}=g_{1} y_{n+k}=z_{n+k}$;

- finally, the point $\tilde{u}$ and its trajectory $\left\{f_{2}^{n} \tilde{u}\right\}$; they are such that $f_{2}^{k} \tilde{u} \approx \tilde{z}=\left(z_{n+k}\right) \approx\left(f_{2}^{n+k} u\right)$.

As we see, the trajectories of points $\tilde{u}$ and $f_{2}^{k} u$ are "always" close to each other. But it is long known that if two trajectories always remain close to each other near a hyperbolic set, then they coincide.

Much of the above needs specification. The most essential critical remark has to do with the first step, the passage from $x$ to $y$. Generally speaking, there are many point in $F_{1}$ that are separated from $f_{1}^{n} x$ by no more than $r$. We do not see reasons to prefer one of these points to all others. Hence, $x \mapsto y$ is a multivalued mapping. This plurality of values is preserved in the next steps until the last one, which leads to $u$, and in this step we would like it to disappear.

Moreover, there are questions of the usual nature. Speaking somewhat vaguely, we mean uniformity of our operations and continuity of their results. They do not require much change, but just accuracy. As for the multivalued mapping $x \mapsto y$, in our problem instead of $x \mapsto y$ we can operate with the inverse mapping $y \mapsto x$, which is one-valued.

We pass to an accurate (but necessarily formalized) exposition of the proof of Theorem 2. Here we use a theorem on families of pseudo-trajectories. Though it is known since late 60's ([1]; see a detailed proof in $[7,10])$, we recall its formulation: It simplifies references where it is used.

\footnotetext{
${ }^{10}$ Often they just speak of a pseudo-trajectory. Such liberty in speech is admissible if the necessary specification of $\delta$ is clear from the context or, if it is not so clear, the necessary specification is given below (especially in preliminary informal discussions).

${ }^{11}$ Which generally consists not of points from $F_{2}$, but of some points close to $F_{2}$.
} 
Definition 1. A family of trajectories (respectively, a family of $\delta$-pseudo-trajectories) of a diffeomorphism $f: M \rightarrow M$ is a triple $(P, \varphi, p)$, where $P$ is a topological space, $\varphi$ is its homeomorphism onto itself, and $p$ is a mapping $P \rightarrow M$ such that $f p=p \varphi$ (respectively, $\rho(f p x, p \varphi x) \leq \delta$ for all $x \in P$ ).

We note that in the case of a family of trajectories $(P, \varphi, p)$ the mapping $p$ is equivariant (with respect to $\varphi$ and $f$ ) and that $p \varphi^{n} x=f^{n} p x$. In the case of a family of $\delta$-pseudo-trajectories there hold inequalities $\rho\left(f p \varphi^{n} x, p \varphi^{n+1} x\right) \leq \delta$, since an arbitrary point $x$ in the inequality $\rho(f p x, p \varphi x) \leq \delta$ can be replaced by $\varphi^{n} x$.

We often say in the sequel (as we have just said) that for two sequences of points $u=\left(u_{n}\right), v=\left(v_{n}\right)$ for all $n$ there holds inequality $\rho\left(u_{n}, v_{n}\right) \leq \alpha$ with some $\alpha$. In this connection we can supply $M^{\mathbb{Z}}$ with a metric $d(u, v)=\sup _{n} \rho\left(u_{n}, v_{n}\right)$ and say that $d(u, v) \leq \alpha$. (In fact, the metric $d$ is used only for sequences such that their elements are close to $F$ or $F_{i}$, but it is most easy to define it on the whole $M^{\mathbb{Z}}$.) If $d(u, v) \leq \alpha$, we say that sequences $u$ and $v$ trace (up to $\alpha, \alpha$-trace) each other. Similarly, in the conclusion of the theorem on families of pseudo-trajectories one can speak of mutual $\alpha$-tracing of families $(P, \varphi, p)$ and $(P, \varphi, q)$.

The theorem on families of pseudo-trajectories asserts that if $F$ is a hyperbolic set of a diffeomorphism $f: M \rightarrow M$ ("one F-situation"), there exist such a neighborhood $U$ of the set $F$ and such a $e(r)>0$ for each $r>0$ that for each family of $e(r)$-pseudo-trajectories ${ }^{12}$ for $(P, \varphi, p)$ such that $p P \subset U$ ("the family lies in $U$ "), there exists a family of trajectories $(P, \varphi, q)$ such that $\rho(p x, q x) \leq r$ for all $x \in P$.

Take the set $F^{\mathbb{Z}}$ such that its elements are bilateral infinite sequences $x=\left(x_{n} ; n \in \mathbb{Z}\right)$ of points in $F$. We supply $F^{\mathbb{Z}}$ with the Tikhonov topology of an infinite product (in our case it is compact, since its factors are). Define in $F^{\mathbb{Z}}$ a Bernoulli shift $\beta$, which takes $x=\left(x_{n}\right)$ to $y=\left(y_{n}\right)$, where $y_{n}=x_{n+1}$ for all $n \in \mathbb{Z}$. Denote by $P(\delta)$ the subset of $F^{\mathbb{Z}}$ consisting of $\left(x_{n}\right)$ such that $\rho\left(x_{n+1}, f x_{n}\right) \leq \delta$ for all $n \in \mathbb{Z}$. The subset $P(\delta)$ is compact, since each condition $\rho\left(x_{n+1}, f x_{n}\right) \leq \delta$ defines a closed subset of the compact set $F^{\mathbb{Z}}$. Moreover, $P(\delta)$ is invariant with respect to $\beta$ and $\beta^{-1}$. It follows from the fact that the countable system of conditions $\rho\left(x_{n+1}, f x_{n}\right) \leq \delta$ is equivalent to the system of the same conditions on $y_{n}=x_{n+1}$ or on $z_{n}=x_{n-1}$. (For example, if we rewrite the $k$ th condition $\rho\left(y_{n+1}, f y_{n}\right) \leq \delta$ as a condition on $x_{n}=y_{n-1}$, it becomes the $(k+1)$ th condition $\rho\left(x_{n+1}, f x_{n}\right) \leq \delta$.) Further under $\sigma$ we mean $\left.\beta\right|_{P(r)}$. Finally, we denote by $p$ the mapping $P(\delta) \rightarrow M$ such that it takes the sequence $y=\left(y_{n}\right)$ into $p y=y_{0}$. Check that $(P(\delta), \sigma, p)$ is a family of $\delta$-trajectories. For $y=\left(y_{n}\right)$ we have: $p y=y_{0}$; $p \sigma y=z_{0}$, where $z=\sigma y$, i.e., $z_{n}=y_{n+1}$, so that $p \sigma y=z_{0}=y_{1}$; hence, $\rho(f p y, p \sigma y)=\rho\left(f y_{0}, y_{1}\right) \leq \delta$ (a special case of inequality $\rho\left(f y_{n}, y_{n+1}\right) \leq \delta$ for $n=0$ ).

Application of the theorem on families of pseudo-trajectories to $(P(\delta), \sigma, p)$ gives us a neighborhood $U \supset F$ and an $e(r)>0$ such that if $p P(\delta) \subset U$ and $\delta \leq e(r)$, there exists a family of trajectories $(P(\delta), \sigma, q)$, which $r$-traces $(P(s), \sigma, p)$. Now condition $p P(\delta) \subset U$ holds automatically, since sequences $y=\left(y_{n}\right) \in P(\delta)$ consist of points of $F$ and therefore $p y=y_{0} \in F \subset U$. Hence, for $m=\min (D / 3, R)$ there exists a family of trajectories $(P(e(m)), \sigma, q)=(P(e(\min (D / 3, R)), \sigma, q)$, which traces the family of trajectories $(P(e(m)), \sigma, p)=(P(e(\min (D / 3, R)), \sigma, p)$ up to $m$.

Lemma 2. If $y, z \in P(e(m))$ (with the same $m=\min (D / 3, R)$ ), then $q y=q z$. (Thus the mapping $q$ is locally constant in the topology defined by the metric $d$. It has even a stronger property: $q$ is constant on each ball of radius $D / 6$ in the metric d.)

Proof. Firstly, $q y$ and $q z$ lie in $S$, since for $y=\left(y_{n}\right) \rho(q y, F) \leq \rho(q y, p y)$ (because $p y=y_{0} \in F$ ), and the last $\rho \leq R$, so that $y \in U_{R}(F) \subset S$. Not only $y$, but all $f^{n} q y=q \sigma^{n} y$ also belong to $S$. Thus, $q y \in I(S)$; similarly, $q z \in I(S)$. Secondly,

$$
P(q y, q z) \leq \rho(q y, p y)+\rho(p y, p z)+\rho(p z, q z) \leq 3 \cdot \frac{D}{3}=D .
$$

\footnotetext{
${ }^{12}$ The letter $e$ stands for the word error. We mean the error made on a single step of the process of calculating the trajectory $f^{n} x$, if we take $x_{n+1}$ instead of $f x_{n}$.
} 
The first and the third term in the sum of three $\rho$ 's do not exceed $m=\max (D / 3, R) \leq D / 3$. The second term equals $\rho\left(y_{0}, z_{0}\right) \leq d(y, z) \leq D / 3$ for $y=\left(y_{n}\right)$, Replacing $y$ and $z$ with $\sigma^{n} y$ and $\sigma^{n} z$ (these are also elements of the same $P(e(m))$ and $d\left(\sigma^{n} y, \sigma^{n} y, \sigma^{n} z\right)=d(y, z) \leq D / 3$ ), we get that $q \sigma^{n} y, q \sigma^{n} y, q \sigma^{n} z \in S$ and $\rho\left(q \sigma^{n} y, q \sigma^{n} z\right) \leq D / 3$. But $q \sigma^{n} y=f^{n} q y, q \sigma^{n} z=f^{n} q z$. other words, $q y, q z \in I(S)$ and all $\rho\left(f^{n} q y, f^{n} q z\right) \leq D$. Due to separation of motions in $S$ with a separation constant $D$, this implies that $q y=q z$.

Fix a compact neighborhood $N$ in $M$. Denote by $\omega(r)$ the modulus of continuity of the mapping $\left.f\right|_{N}: N \rightarrow M$. Show that if $x \in I U_{r}(F) \subset U_{r}(F) \subset N, y_{n} \in F$, and $\rho\left(f^{n} x, y_{n}\right) \leq r$ for all $n \in \mathbb{Z}$, then the sequence $y=\left(y_{n}\right)$ is a $\delta$-trajectory with $\delta=\delta(r)=r+\omega(r)$. In fact,

$$
\rho\left(f y_{n}, y_{n+1}\right) \leq \rho\left(f y_{n}, f f^{n} x\right)+\rho\left(f^{n+1} x, y_{n+1}\right) \leq \omega\left(\rho\left(y_{n}, f^{n} x\right)\right)+r \leq \omega(r)+r .
$$

Reducing $R$ and $D$ if necessary, we can assume that $U_{R}(F) \subset N$ and that for $\bar{m}=\min (R, D / 2)$ there holds the inclusion $U_{\bar{m}} \subset N$. These neighborhoods are assumed closed and hence compact. (This implies compactness of other neighborhoods that we encounter below.) For the sake of brevity, add to the notation $\bar{m}$ three more notations: $\bar{e}=r(\bar{m})$, we denote $r$ such that $\delta(r)=r+\omega(r)=\bar{e}$ by $\bar{r}$, and $\delta(\bar{r})$ by $\bar{\delta}$. Mapping $q: P(\bar{\delta}) \rightarrow M$ allows us to a certain degree to replace trajectories $\left\{f^{n} x\right\}$ close to $F$ with sequences $y \in P(\bar{\delta})$ such that $q y=x$.

But $P(\bar{\delta})$ contains many sequences $y=\left(y_{n}\right)$ not needed for our purposes. We have the "smallness parameter" $r$ such that its absolute value ("smallness degree") can be specified only later. Firstly, we need only sequences $y=\left(y_{n}\right)$ such that $q y \in I U_{r}(F)$, and concerning $y$ in $P(\bar{\delta})$ we know only that $q y \in I U_{\bar{m}}(F)$ (since $\bar{\delta}=e(\bar{m}) \Rightarrow \rho\left(f^{n} q y, p \sigma^{n} y\right)=\rho\left(q \sigma^{n} y, p \sigma^{n} y\right) \leq \bar{m}$, and $\left.p \sigma^{n} y=y_{n} \in F\right)$. And secondly, we want the trajectory $\left\{f^{n} q y\right\}=\left\{q \sigma^{n} y\right\}$ to $r$-trace the sequence $\left(p \sigma^{n} y\right)=\left(y_{n}\right)$, but generally we know only that $\rho\left(q \sigma^{n} y, p \sigma^{n} y\right) \leq \bar{m}$. Removing from $P(\bar{\delta})$ sequences we do not need, we pass to the set

$$
Q(r)=\left\{y \in P(\delta(r)): \forall n \in \mathbb{Z} \quad \rho\left(p \sigma^{n} y, q \sigma^{n} y\right) \leq r\right\},
$$

assuming that $r \leq \bar{r}$. At first sight (18) does not quite comply with the above, but this discrepancy is only apparent, because:

(a) if $x \in I U_{r}(F)$ and $y=\left(y_{n}\right) \in F^{\mathbb{Z}}$ are such as above ${ }^{13}$, then $y \in Q(r)$ and $x=q y($ for $P(\delta(r)) \subset$ $P(\bar{\delta})$ we may speak about $q y)$;

(b) conversely, if $y=\left(y_{n}\right) \in Q(r)$ and $x=q y$, then $x \in I U_{r}(F)$ and all $\rho\left(f^{n} x, y_{n}\right) \leq r$.

(In particular, (a) and (b) imply $q Q(r)=I U_{r}(F)$.) We have already seen that if $x \in I U_{r}(F)$, all $y_{n} \in F$, and $\rho\left(f^{n} x, y_{n}\right) \leq r$, then $y=\left(y_{n}\right)$ is a $\delta(r)$-pseudo-trajectory, so that in fact $y \in Q(r)$.

Since $\rho\left(f^{n} x, y_{n}\right) \leq r \leq R$ and $y_{n} \in F$, we have $f^{n} x \in U_{R}(F) \subset S$. And since $\rho\left(f^{n} q y, y_{n}\right)=$ $\rho\left(q \sigma^{n} y, p \sigma^{n} y\right) \leq \bar{m} \leq R$, we have $f^{n} q y \in U_{R}(F) \subset S$. Finally, using the fact that $\rho\left(y_{n}, f^{n} q y\right)=$ $\rho\left(p \sigma^{n} y, q \sigma^{n} y\right) \leq \bar{D} / 2$, we find

$$
\rho\left(f^{n} x, f^{n} q y\right)=\rho\left(f^{n} x, y_{n}\right)+\rho\left(y_{n}, f^{n} q y\right) \leq r+\frac{D}{2} \leq 2 \frac{D}{2}=D .
$$

Due to separation of motions in $S$, we get that $x=q y$. This proves (a).

Now let $y \in Q(r)$ and $x=q y$. Then

$$
\rho\left(f^{n} x, y_{n}\right)=\rho\left(\sigma^{n} q y, p \sigma^{n} y\right)=\rho\left(q \sigma^{n} y, p \sigma^{n} y\right) \leq r
$$

(the last inequality is explicitly contained in the definition of $Q(r)$ ). We have proved the inequality in (b), and it implies as usual that $x \in I U_{r}(F)$. Thus, (b) is also proved.

9. Now we pass to the "two- $F$ situation," and in particular we have to deal with "one- $F$ situation" where $M_{i}, f_{i}, F_{i}$ are taken for $M, f, F$. Then the objects introduced while discussing the "one- $F$ situation" will be marked by the subscript $i$. Thus, $R_{i}, D_{i}, S_{i}, \omega_{i}, l_{i}, \bar{m}_{i}, \delta_{i}, P_{i}\left(\delta_{i}\right), p_{i}$, and $q_{i}$ appear.

\footnotetext{
${ }^{13}$ Such $y_{n} \in F$ do exist, since $\left.\rho\left(f^{n} x, F\right) \leq F\right) \leq r$ for all $n$.
} 
The mapping $g_{1}: F_{1} \rightarrow F_{2}$ can be applied to elements of the sequence $y=\left(y_{n}\right) \in F_{1}^{\mathbb{Z}}$, in particular, to elements $Q(\delta)$. Thus we obtain a sequence $\left(g_{1} y_{n}\right) \in F_{2}^{\mathbb{Z}}$ denoted by $\hat{g}_{1} y$. The mapping $\hat{g}_{1}$ takes $P_{1}(\delta)$ to a part of some $P_{2}(\Delta)$ where $\Delta$ is characterized by the modulus of continuity $\Omega(r)$ of the mapping $g_{1}$. Namely,

$$
\rho\left(f_{2} g_{1} y_{n}, g_{1} y_{n+1}\right)=\rho\left(g_{1} f_{1} y_{n}, g_{1} y_{n+1}\right) \leq \Omega\left(\rho\left(f_{1} y_{n}, y_{n+1}\right)\right) \leq \Omega(\delta) .
$$

Therefore,

$$
\hat{g}_{1} P_{1}\left(\delta_{1}\right) \subset P_{2}\left(\Omega\left(\delta_{1}\right)\right) .
$$

Lemma 3. For $r$ small enough, one has:

(a) $\delta_{1}(r) \leq e_{1}\left(\bar{m}_{1}\right)$, so that the mapping $q_{1}$ is defined on $Q_{1}\left(\delta_{1}\right)$;

(b) $q_{1} Q_{1}\left(\delta_{1}(r)\right)=I U_{r}\left(F_{1}\right)$;

(c) $\Omega\left(\delta_{1}\right) \leq e_{2}\left(\bar{m}_{2}\right)$ so that the mapping $q_{2}$ is defined on the set $P_{2}(\Delta)$ where $\Delta=\Omega\left(\delta_{1}\right)$, and, in particular, on $\hat{g}_{1} Q_{1}(\delta)$;

(d) if $y, z \in Q_{1}(r)$ and $q_{1} y=q_{1} z$, then $q_{2} \hat{q}_{1} y=q_{2} \hat{q}_{1} z$.

Items (a), (b), (c) only sum up some part of the above. We prove (d). As follows from Lemma 2, it suffices to establish that for $r$ small enough the following statement holds:

if $y=\left(y_{n}\right), z=\left(z_{n}\right) \in Q_{1}(r)$ and $q_{1} y=q_{1} z$, then

$$
\begin{aligned}
& \hat{q}_{1} y, \hat{q}_{1} z \in P_{2}\left(e_{2}\left(\bar{m}_{2}\right)\right) \quad\left(\text { where } \bar{m}_{2}=\min \left(R_{2}, D_{2} / 3\right)\right), \\
& \left.\hat{d}\left(\hat{g}_{1} y, \hat{g}_{1} z\right) \leq D_{2} / 3 \quad \text { (i.e., } \forall n \quad \rho\left(g_{1} y_{n}, g_{1} z_{n}\right) \leq D_{2} / 3\right) .
\end{aligned}
$$

From the fact that $y, z \in Q_{1}(r)$ it follows (see (19)) that $\hat{g}_{1} y, \hat{g}_{1} z \in P_{2}\left(\Omega\left(\delta_{1}\right)\right.$ ). Hence, if $r$ is so small that $\Omega\left(\delta_{1}(r)\right) \leq e_{2}\left(\bar{m}_{2}\right)$, then we have (20). Further, for $x=q_{1} y=q_{1} z$

$$
\rho\left(y_{n}, z_{n}\right) \leq \rho\left(y_{n}, f^{n} x\right)+\rho\left(f^{n} x, z_{n}\right) \leq r+r=2 r,
$$

therefore, $\rho\left(g_{1} y_{n}, g_{1} z_{n}\right) \leq \Omega\left(\rho\left(y_{n}, z_{n}\right)\right) \leq \Omega(2 r)$ and $(21)$ will be guaranteed if (in addition to the previous assumptions on $r$ ) we require that $\Omega(2 r) \leq D_{2} / 3$.

Conclusion of the proof of Theorem 2. Define a mapping $h_{1}: I U_{r}\left(F_{1}\right) \rightarrow M_{2}$ in the following way (assuming $r$ as small as needed for validity of the statements we use). Let $x \in I U_{r}(F)$. To define $h_{1}(x)$, take some $y \in Q_{1}(r)$ such that $q_{1}(y)=x$ and put $h_{1}(x)=q_{2} \hat{g}_{1} y$. The correctness of this definition follows from Lemma 3: If instead of $y$ we take in $Q_{1}(r)$ another element $z$ such that $q_{1} z=x$, then we get $q_{2} \hat{g}_{1} z=q_{2} \hat{g}_{1} y$. The mapping $h_{1}$ constructed this way is equivariant: if $q_{1} y=x$, then $q_{1} \sigma^{n} y=f_{1}^{n} q y=f_{1}^{n} x$ and

$$
h_{1} f_{1}^{n} x=q_{2} \hat{g}_{1} \sigma^{n} y=q_{2} \sigma^{n} \hat{g}_{1} y=f_{2}^{n} q_{2} \hat{g}_{1} y=f_{2}^{n} h_{1} x .
$$

(Here we use obvious equalities $\hat{g}_{1} \sigma^{n}=\sigma^{n} \hat{g}_{1}$ and $q_{2} \sigma^{n}=f_{2}^{n} q_{2}$.) Finally, the continuity of $h_{1}$ immediately follows from

Lemma 4. Let $A, B, C$ be Hausdorff spaces, $A$ compact, $f: A \rightarrow B, g: A \rightarrow C$ continuous mappings, $f$ surjective, and

$$
\forall a, a^{\prime} \in A \quad f a=f a^{\prime} \Rightarrow g a=g a^{\prime},
$$

so that we can correctly define the mapping $h: B \rightarrow C$ putting $h b=g a$ with an arbitrary $a \in f^{-1} b$. Then $h$ is continuous.

Proof. We must prove that if $U$ is an open subset of $C$, then $h^{-1} U$ is an open subset of $B$. Since $\{U, C \backslash U\}$ is a disjunctive partition of $C$, we have that $\left\{h^{-1} U, h^{-1}(C \backslash U)\right\}$ is a disjunctive partition of $B$. Hence it suffices to establish that $h^{-1}(C \backslash U)$ is closed.

For any $D \subset C$ its preimage $h^{-1} D=f g^{-1} D$, since

$$
b \in h^{-1} D \Leftrightarrow h b \in D \Leftrightarrow \exists a \in A \quad f a=b, g a \in D \Leftrightarrow \exists a \in A \quad f a=b, a \in g^{-1} D \Leftrightarrow f g^{-1} D \ni b .
$$


In particular, $h^{-1}(C \backslash U)=f g^{-1}(C \backslash U)$. Since $U$ is open, $C \backslash U$ is closed, then so is $g^{-1}(C \backslash U)$. The latter set, being a closed subset of the compact set $A$, is compact. Its image $f^{-1}(C \backslash U)$ under the continuous mapping $f$ is also compact, and therefore it is a closed subset of $B$.

10. Finally we start proving Theorem 3 . Here we will have the one- $F$ situation and use the respective notation.

Lemma 5. Under the assumptions of Theorem 3, for any $\delta>0, F$ has a neighborhood $W(\delta)$ in $M$ such that $W(\delta) \subset U$ (hence, $\varphi$ is defined everywhere on $I(W(\delta)))$ and $\rho(x, \varphi x) \leq \delta$ for all $x \in I(W(\delta))$.

Proof. The function $x \mapsto \rho(x, \varphi x)$ is continuous on $I(U)$ and vanishes on $F$. Hence $F$ has a neighborhood $G(\delta)$ in $M$ such that if $x \in I(U) \cap G(\delta)$, then $\rho(x, \varphi x) \leq \delta$. By Lemma $1, F$ has a neighborhood $W(\delta)$ in $M$ such that $W(\delta) \subset U$ and $I(W(\delta)) \subset I(U) \cap G(\delta)$. Hence if $x \in I(W(\delta))$, then $\varphi x$ is defined and $\rho(x, \varphi x) \leq \delta$. As we can see, $W(\delta)$ has all the properties required in the formulation of the Lemma.

Proof of Theorem 3. It suffices to check the existence of a neighborhood $V$ of the set $F$ in the space $M$ such that $V \subset U$ and

$$
x \in I V \Rightarrow \text { all } f^{n} x, f^{n} \varphi x \in S \text { and } \rho\left(f^{n} x, f^{n} \varphi x\right) \leq D
$$

(then $x=\varphi x$ due to separation of motions). Using Lemma 5 with $\delta=\min (R / 2, D)$, we get that $F$ has a neighborhood $W(\delta)$ in $M$ with required properties. Further there is $r>0$ such that the $r$-neighborhood $V_{r}(F) \subset W(\delta), 2 r \leq \delta$, and $r \leq D$. This $V_{r}(F)$ can be taken for the required $V$. Since in Lemma $5 W(\delta) \subset U$, we have $V \subset U$. If $x \in I V$, then all $f^{n} x \in V, \rho\left(f^{n} x, F\right) \leq r \leq R$, and $f^{n} x \in U_{R}(F) \subset S$. Further, all $\varphi f^{n} x$ are defined and $\rho\left(f^{n} x, \varphi f^{n} x\right) \leq \delta \leq R / 2$, and $f^{n} \varphi x$ are defined and coincide with $\varphi f^{n} x$ due to equivariance of $\varphi$. Therefore, $\rho\left(f^{n} \varphi x, F\right) \leq \rho\left(\varphi f^{n} x, f^{n} x\right)+\leq \delta \leq R$; hence, $f^{n} \varphi x \in U_{R}(F) \subset S$. Finally, $\rho\left(f^{n} x, f^{n} \varphi x\right)=\rho\left(f^{n} x, \varphi f^{n} x\right) \leq \delta<D$. We have checked the validity of $(23)$.

Acknowledgements. This work was supported by the RFBR grant 11-01-00384, by the grant of the President of the Russian Federation supporting the leading scientific schools NSh-8508.2010.1, and by the program of the Presidium of RAS "Mathematical control theory." The author is thankful to Kleptsyn and Klimenko for their help in the preparation of the manuscript.

\section{REFERENCES}

1. D. V. Anosov, "On a class of invariant sets of smooth dynamical systems," Proc. of V Int. conference on nonlinear oscillations, Kiev, Inst. Math. of Academy of Sciences of Ukrainian SSR, 2, 39-44 (1970).

2. D. V. Anosov, "Extension of null-dimensional hyperbolic sets to locally maximal ones," Sb. Math., 201, 3-17 (2010).

3. D. V. Anosov, "Intrinsic character of one property of hyperbolic sets," J. Dyn. Control Syst., 16, 485-492 (2010).

4. D. V. Anosov, "On some hyperbolic sets," Math. Notes, 87, 650-668 (2010).

5. D. V. Anosov, "Local maximaility of hyperbolic sets," Proc. Steklov Inst., 273, 28-29 (2011).

6. S. Crovisier, "Une remarque sur les ensembles hyperboliques localement maximaux," C. R. Math. Acad. Sci. Paris, 334, 401-404 (2001).

7. A. B. Katok and B. Hasselblatt, Introduction into Modern Theory of Dynamical Systems, Factorial, Moscow (1999).

8. T. Fisher, "Hyperbolic sets that are not locally maximal," Ergodic Theory Dynam. Syst., 26, 1491-1509 (2006).

9. T. Fisher, Transitive hyperbolic sets on surfaces, Preprint, Brigham Young Univ., Provo, UT (2009). 
10. M. Shub, "Stabilité globale des systèmes dynamiques," Astèrisque, 56, Soc. Math. France, Paris (1978).

Dmitry V. Anosov

Steklov Mathematical Institute, 8, Gubkina st., 119991 Moscow, Russia

Lomonosov Moscow State University, GSP-1, Leninskie Gory, 119991 Moscow, Russia

E-mail: anosov@mi.ras.ru 